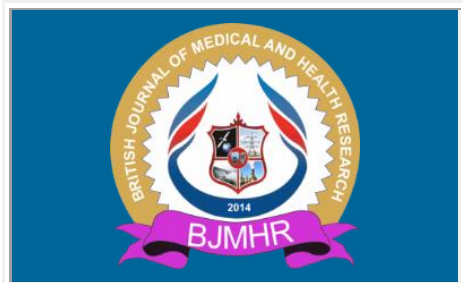

\title{
BJMHR
}

British Journal of Medical and Health Research

Journal home page: www.bjmhr.com

\section{Isoform A of Lactate Dehydrogenase (LDH-A), a potential ultra-early, high sensitivity biomarker of tumorigenesis.}

\author{
Prieto Gratacós ${ }^{1 *}$ E., Aguirre Gauto² P. \\ 1 Precision Metabolic Oncology UK \\ 2 Center for Metabolic Therapy of Buenos Aires
}

\section{ABSTRACT}

A higher than normal percentual contribution of isoform A to the lactate dehydrogenase enzymatic cluster reveals a pathological shift towards fermentative metabolism somewhere within the organism of the host. The hypermetabolic phenotype expressed by tumour cells, a well-documented hallmark of cancer, is rooted on the catalytic action of several enzymes, amongst which hexokinase 2 and LDH-A are key players, supporting cell survival and neoangiogenesis as well as driving tumour growth. The physiological, intrinsic secretion of lactic dehydrogenase A in healthy humans has not been described formally and can be used as a functional frame of reference in the ultra-early detection of neoplastic transformation. In healthy subjects, even with total plasma LDH within the normal range, increases in the isoform A surpassing three or more standard deviations above its mean percentual contribution to the enzymatic cluster suggest a pathological reprogramming of energy metabolism. Herein, preliminary evidence is presented, supporting the notion of LDH-A as a screening tool for ultra-early, actionable detection of microtumours, during the initial or avascular phase of neoplastic progression.

Keywords: Isoenzyme A, lactic dehydrogenase, Warburg effect, metabolic cancer therapy 


\section{INTRODUCTION}

\section{Preliminary considerations}

Depending on the relative concentration of each of its five isoenzymes, lactic dehydrogenase catalyses either the conversion of pyruvic acid into acetyl CoA or its fermentation into lactic acid, thus procuring an energy source for cells unable to extract it by means of oxidative phosphorylation. The implications are vast, and LDH-A's fermentative power has been found to be an intricate part of survival mechanisms in many cancers, including breast, lung, prostate and pancreatic cancer ${ }^{1-5}$.

Fermentative hypermetabolism is a widely recognized hallmark of tumour cells ${ }^{6-8}$. Evidence of ultrastructural mitochondrial pathology (cristodysmorphia) has recently been obtained by means of electron microscopy ${ }^{9}$. This work has provided visual confirmation as well as crucial insights towards a mechanistically explanation of the progressive deterioration of the respiratory quotient (RQ) in neoplastic cells. In vivo, the actual yield of oxidative phosphorylation is approximately $33.45 \mathrm{ATP} / \mathrm{glucose}$, a remarkable efficiency in the harvesting of the metabolic power contained within high energy chemical bonds ${ }^{10}$. Although not as high as the theoretical yield of 36 ATP moles for each mole of glucose sent through the glycolysis/OXPHOS oxidative degradation cascade, the complete process of respiration is about sixteen-fold more efficient than fermentation alone ${ }^{10,11}$. In the absence of properly functioning mitochondria, the energetic needs of anaplastic cells can only be met by low yield/high transaction volume metabolic pathways, such as anaerobic glycolysis, substratelevel phosphorylation and glutaminolysis ${ }^{12}$. Though a striking biological regression from the evolutionary standpoint, fermentation provides a dependable, robust pathway to secure both building blocks and metabolically utilizable energy within anaplastic cells.

\section{Biological implications of plasma isoform $A$ abnormalities}

Initially isolated in Plasmodium falciparum ( $p f \mathrm{LDH})$ as well as in liver and muscle tissue (therefore dubbed "M", also known as LDH5), isoform A of the LDH cluster, provides an alternate pathway to harvesting the energy contained in pyruvate under conditions of overwhelming functional demand ${ }^{13}$. Besides providing a secondary, anaerobic energy source during intense physical exertion, this isoenzyme has no other known functions in healthy organisms ${ }^{14}$. For properly rested individuals (LDH-A has a half-life of 9 hours), increases in plasma levels of this enzyme in excess of three standard deviations are almost certainly due to an increase in malignant fermentative metabolism taking place within anaplastic cells, due to a loss of their respiratory capacity ${ }^{15}$.

For humans in good overall health, more specifically, individuals with no biochemically or clinically discernible tumoural pathology, it is a regular occurrence to find low -i.e. 
physiological- levels of several substances regarded as tumour markers ${ }^{16,17}$. Based on our clinical experience and on general theoretical knowledge we set to consider as clinically significant -requiring further scrutiny- any increase of LDH-A greater than 2 standard deviations above the mean plasma concentration of healthy subjects. Such consideration stems from the fact that isoform $\mathrm{A}$ is found as a "constitutive secretion" in the blood of healthy individuals under 53 years of age in concentrations that rarely exceed $10 \mathrm{ng} / \mathrm{ml}$.

Immunohistochemical techniques have demonstrated that it is LDH-A -but not other isoenzymes within the family- that is predominantly expressed in neoplastic tissues ${ }^{18}$. Isoform A of the LDH family can, therefore, be regarded as an early biomarker for highly glycolytic malignancies. Our group and many others have found elevated serum LDH-A in virtually all cancer patients tested, regardless of tissue origin, age, disease stage or previous treatment ${ }^{19-22}$.

\section{Eligibility criteria for healthy subjects providing reference values}

Voluntary participants were required to be free of any apparent illness, without any history of previous oncological disease, and to be younger than 53 years of age. The upper cut-off value for total lactic dehydrogenase (LDH) was set at $200 \mathrm{U} / \mathrm{L}$, placing every participant comfortably within the normal physiological range reported by regional laboratories ${ }^{23}$. Subjects with a history of exertional myoglobinuria, hinting at an inborn error of the lactic dehydrogenase pathway, were also not included.

\section{Patients, Materials and Methods}

Thirty healthy volunteers, 18 females, 12 males, with ages ranging from 24 to 52 years $(\bar{x} 33$, $\hat{x} 32$ ), were enrolled amongst healthcare professionals and software engineers to provide blood samples. Additionally, 28 patients with a confirmed diagnosis of cancer -in a spectrum of tumoral pathologies including cancers to the breast $(21.4 \%)$, colon $(14.3 \%)$, prostate $(7.1 \%)$, uterus $(10.7 \%)$, ovary $(7.1 \%)$, liver $(7.1 \%)$, lung (3.6\%), kidney $(3.6 \%)$, as well as exocrine pancreatic cancer $(7.1 \%)$, glioblastoma (10.7\%), sarcoma (3.6\%), and chondroblastoma (3.6\%)- were also analyzed for total LDH and the A fraction.

For all participants, liver and kidney functions (Chemical Analyzer A15, BIOSYSTEMS), as well as hematopoietic status (Haematology Cell Counter Advia 560, SIEMENS), were analyzed in order to assess their overall physiological condition and establish a baseline. Blood specimens were obtained after eight hours of their last meal and twelve hours without any physical exertion. Participants were asked to arrive at our laboratory facilities by automobile, avoiding physically demanding means of locomotion such as climbing stairs, long walks or riding a bicycle. Throughout the process of acquiring the blood samples, technicians were careful not to strap the patients' selected limb, nor allow them to forcefully make a fist to engorge the blood vessels. These common practices, aimed at improving accessibility to the veins in the upper extremities, are known to artificially increase total LDH in the sample. LDH- 
A was determined by means of Enzyme-Linked Immunosorbent Assay (Wuhan Fine Biotech, ELISA kit). Blood samples were heparinised and centrifuged at $1.600 \mathrm{rpm}$ for 10 minutes, then processed according to manufacturer specifications. Written informed consent was obtained from each healthy volunteer and cancer patient. All participants and their close relatives were previously instructed in every instance on the necessary preparations and precautions.

\section{Rationale for age exclusion criteria}

Cancer incidence in the population has been uniformly found to increase as a function of age (24-27). A similar trend has been demonstrated regarding all-cause mortality, with the probability of death doubling every eight years from puberty onwards ${ }^{28,29}$. First reported by mathematician and actuarian Benjamin Gompertz, this observation about the doubling time of the statistical probability of dying stands unchallenged today ${ }^{30}$. Several authors have independently validated the Gompertz equation as a tool for modelling tumour growth ${ }^{31-33}$. Consequently, it stands to reason that the probability of bearing an imperceptible, subclinical neoplastic pathology that could contribute to plasma LDH-A levels increases exponentially in direct proportion with chronological age. Limiting to 53 years the age of the healthy subjects included in the construction of a normal distribution for isoform $\mathrm{A}$ is intended to filter out individuals that could inadvertently carry an LDHA-secreting tumour, thus allowing for a more sensitive biomarker. This was a conscious decision on our part, intended to strongly enhance the sensitivity of the test, even at the expense of a marginal decrease in specificity.

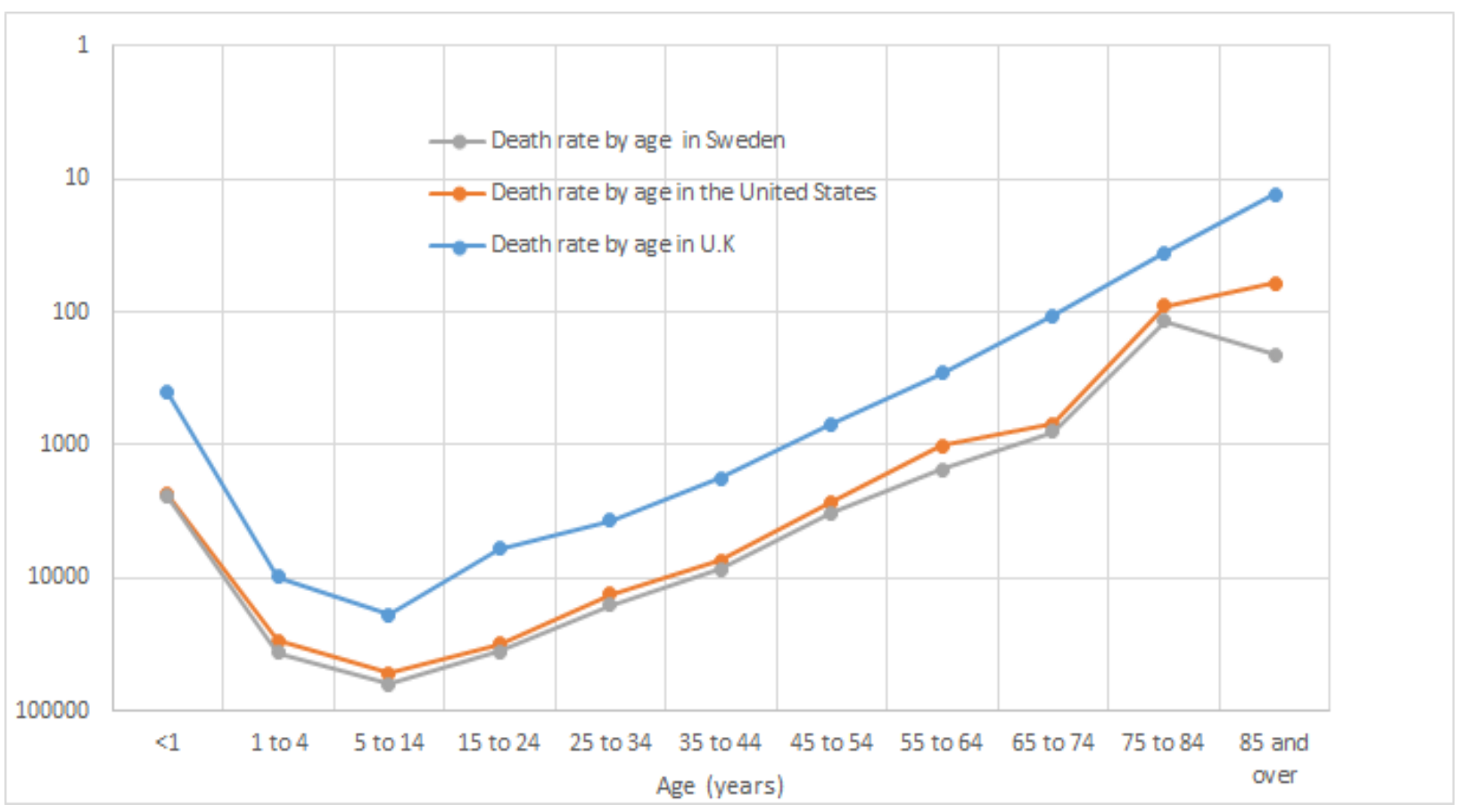

Figure 1. A demonstration of Gompertz's law through a semi-log chart of all-cause mortality and age trajectories of Sweden, UK and USA populations (excerpted and combined from references 34, 35 and 36). Across many independent studies, the 
logarithm of the total death rate and the death rates for some individual diseases are linear functions of the chronological age, consistently doubling every $\sim 8.4$ years.

\section{RESULTS AND DATA ANALYSIS}

In our set of 30 healthy volunteers, plasma levels of LDH-A were found to range from 4.3 $\mathrm{ng} / \mathrm{ml}$ to $10.0 \mathrm{ng} / \mathrm{ml}(\bar{x} 6.84 ; \hat{x} 6.9 ; \sigma 1.48)$. The Confidence Interval $95 \%$ was estimated to be 6.39 -- 7.29. In this set of healthy subjects, the percentual contribution of isoform A to total LDH ranged from $1.7 \%$ to $4.8 \%(\bar{x} 2.79, \sigma 0.7)$.

Amongst the 28 cancer patients, LDH-A was universally elevated, ranging from $15.0 \mathrm{ng} / \mathrm{ml}$ to $51.1 \mathrm{ng} / \mathrm{ml}(\bar{x} 30.8 ; \hat{x} 31.0 ; \sigma 6.07)$. Relative to the mean of healthy subjects, plasma levels of LDH-A were pronouncedly increased in all cancer patients. Furthermore, the percentual contribution of isoenzyme A to total LDH was also higher than the healthy mean (that is, $\geq 2.8$ $\%$ ) in $96.5 \%$ of the cases. However, patients $\mathrm{N}^{\circ} 27$ and $\mathrm{N}^{\circ} 1$-whose percentual contributions fell within or in close vicinity to normal ranges- had unmistakably pathological total LDH levels (703 U/L and $912 \mathrm{U} / \mathrm{L}$, respectively). On average, the percentual contribution of isoform $\mathrm{A}$ in tumour-bearing patients was $8.3 \%$ (2.8 -- 15.9).

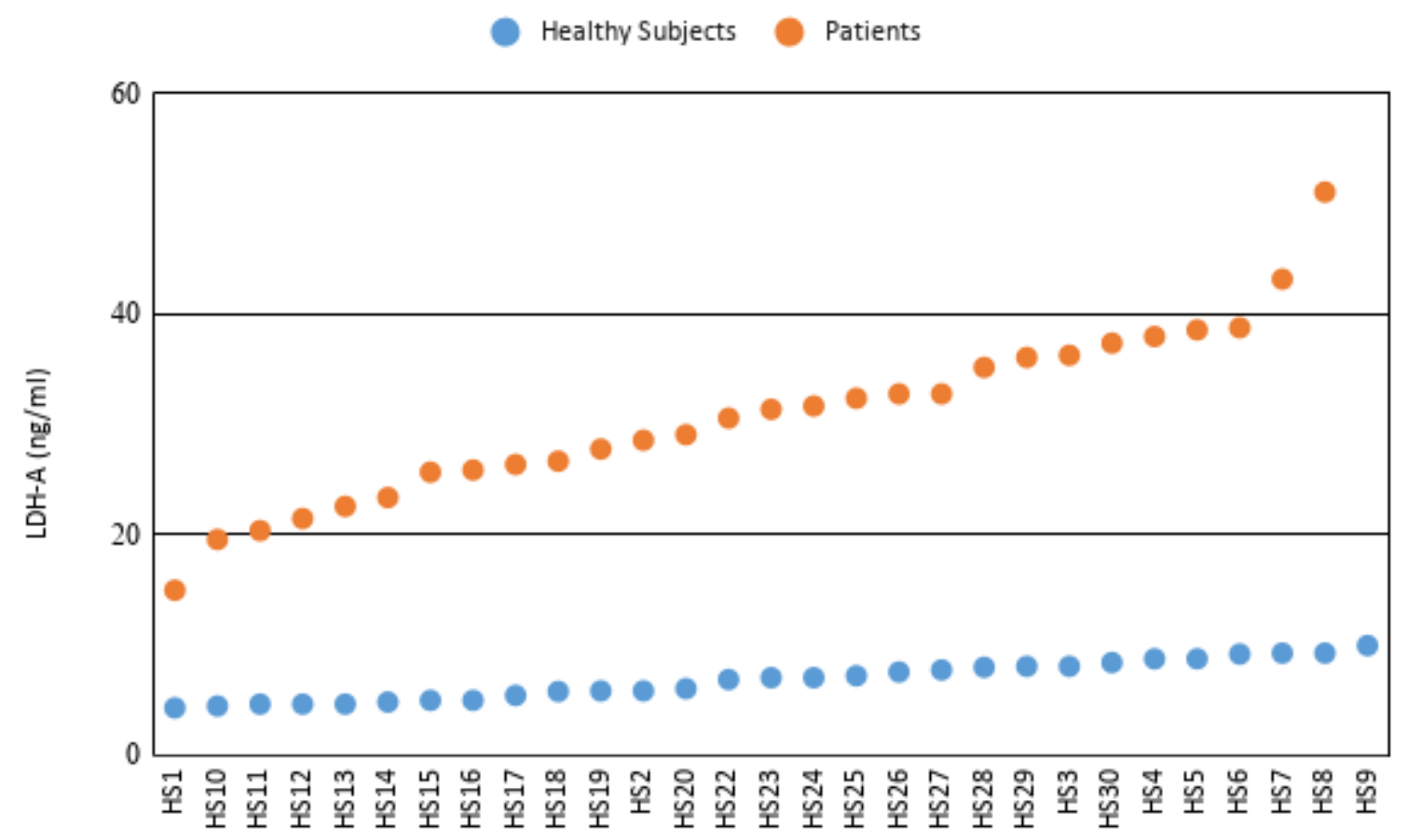

Figure 2: Isoenzyme LDH-A levels of 30 healthy subjects under 53 years of age (blue) and 28 cancer patients (orange), arrayed in ascending order. 


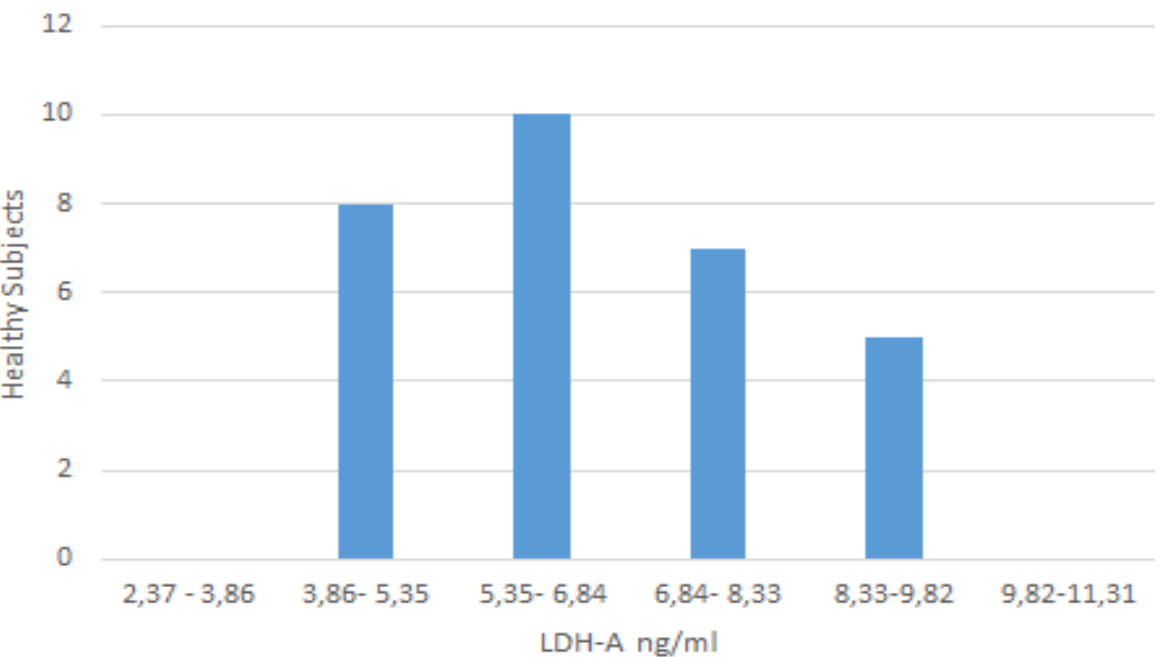

Figure 3: Distribution of isoenzyme LDH-A in healthy subjects $(n=30)$ showing a rightward skew. Mean plasma level: $6.84 \mathrm{ng} / \mathrm{ml}$, standard deviation: $1.48 \mathrm{ng} / \mathrm{dl}$.

Table 1: Total LDH, isoenzyme A and percentual contribution of iso-A to total enzymatic cluster amongst healthy subjects $(n=30)$.

\begin{tabular}{|c|c|c|c|c|c|c|c|}
\hline $\begin{array}{l}\text { Healthy } \\
\text { subjects }\end{array}$ & $\begin{array}{l}\text { LDH- } \\
\text { A }\end{array}$ & $\begin{array}{l}\text { LDH- } \\
\text { A }\end{array}$ & $\begin{array}{l}\text { Total } \\
\text { LDH }\end{array}$ & $\begin{array}{l}\text { Ratio } \\
\text { Total/iso A }\end{array}$ & $\begin{array}{l}\text { Percentual } \\
\text { contribution }\end{array}$ & Age & Gender \\
\hline & $(\mathrm{ng} / \mathrm{ml})$ & (U/L) & (U/L) & & $\%$ & & \\
\hline HS1 & 4,7 & 3,1 & 165,0 & 53,0 & 1,9 & 27 & $\mathrm{~F}$ \\
\hline HS2 & 7,2 & 4,8 & 177,0 & 36,7 & 2,7 & 35 & M \\
\hline HS3 & 4,7 & 3,1 & 172,0 & 55,3 & 1,8 & 36 & $\mathrm{~F}$ \\
\hline HS4 & 5,0 & 3,3 & 192,0 & 57,5 & 1,7 & 33 & M \\
\hline HS5 & 8,8 & 5,8 & 167,0 & 28,5 & 3,5 & 35 & M \\
\hline HS6 & 6,9 & 4,6 & 171,0 & 37,2 & 2,7 & 34 & $\mathrm{~F}$ \\
\hline HS7 & 8,4 & 5,6 & 161,0 & 28,6 & 3,5 & 32 & $\mathrm{~F}$ \\
\hline HS8 & 7,1 & 4,7 & 177,0 & 37,6 & 2,7 & 28 & F \\
\hline HS9 & 8,8 & 5,8 & 136,0 & 23,2 & 4,3 & 30 & M \\
\hline HS 10 & 9,3 & 6,2 & 143,0 & 23,1 & 4,3 & 35 & M \\
\hline HS11 & 5,8 & 3,8 & 135,0 & 35,1 & 2,8 & 27 & $\mathrm{~F}$ \\
\hline HS12 & 7,6 & 5,0 & 138,0 & 27,6 & 3,6 & 26 & $\mathrm{~F}$ \\
\hline HS 13 & 9,2 & 6,1 & 128,0 & 21,1 & 4,8 & 30 & M \\
\hline HS 14 & 5,5 & 3,6 & 145,0 & 40,2 & 2,5 & 27 & F \\
\hline HS 15 & 5,9 & 3,9 & 139,0 & 35,9 & 2,8 & 25 & M \\
\hline HS16 & 4,7 & 3,1 & 141,0 & 45,7 & 2,2 & 25 & M \\
\hline HS17 & 6,1 & 4,0 & 173,0 & 43,1 & 2,3 & 26 & $\mathrm{M}$ \\
\hline HS 18 & 8,0 & 5,3 & 167,0 & 31,6 & 3,2 & 26 & $\mathrm{~F}$ \\
\hline HS19 & 4,9 & 3,2 & 154,0 & 48,0 & 2,1 & 25 & $\mathrm{~F}$ \\
\hline HS20 & 5,0 & 3,3 & 171,0 & 51,6 & 1,9 & 33 & $\mathrm{~F}$ \\
\hline HS21 & 7,8 & 5,1 & 187,0 & 36,4 & 2,7 & 19 & $\mathrm{~F}$ \\
\hline HS22 & 5,9 & 3,9 & 168,0 & 43,3 & 2,3 & 26 & $\mathrm{~F}$ \\
\hline HS23 & 4,3 & 2,9 & 163,0 & 57,0 & 1,8 & 25 & F \\
\hline HS24 & 7,1 & 4,7 & 129,0 & 27,4 & 3,7 & 43 & $\mathrm{~F}$ \\
\hline HS25 & 8,1 & 9,6 & 214,0 & 22,2 & 4,5 & 52 & $\mathrm{M}$ \\
\hline HS26 & 6,6 & 4,4 & 191,0 & 43,7 & 2,3 & 43 & M \\
\hline HS27 & 10,0 & 6,6 & 169,0 & 0,7 & 3,9 & 45 & F \\
\hline HS28 & 9,3 & 6,2 & 159,0 & 25,7 & 3,9 & 41 & $\mathrm{~F}$ \\
\hline HS29 & 4,5 & 3,0 & 141,0 & 47,0 & 2,1 & 52 & $\mathrm{M}$ \\
\hline HS30 & 8,1 & 5,3 & 156,0 & 29,2 & 3,4 & 51 & $\mathrm{~F}$ \\
\hline
\end{tabular}


Table 2: Total LDH, isoform A and percentual contribution of iso-A to total enzymatic cluster amongst patients with a confirmed diagnosis of tumoural pathology $(n=28)$.

\begin{tabular}{|c|c|c|c|c|c|c|c|c|}
\hline Patients & $\begin{array}{l}\text { LDH- } \\
\text { A }\end{array}$ & $\begin{array}{l}\text { LDH- } \\
\text { A }\end{array}$ & $\begin{array}{l}\text { Total } \\
\text { LDH }\end{array}$ & $\begin{array}{l}\text { Ratio } \\
\text { Total/iso A }\end{array}$ & $\begin{array}{l}\text { Percentual } \\
\text { contribution }\end{array}$ & Age & Subtype & $\begin{array}{l}\text { Gen } \\
\text { der }\end{array}$ \\
\hline & (ng/ml) & (U/L) & (U/L) & & $\%$ & & & \\
\hline Patient 1 & 38,8 & 25,8 & 912,0 & 35,3 & 2,8 & 59,0 & Colon & $\mathrm{M}$ \\
\hline Patient 2 & 32,8 & 21,8 & 335,0 & 15,4 & 6,5 & 62,0 & Liver & M \\
\hline Patient 3 & 27,8 & 18,4 & 373,0 & 20,3 & 4,9 & 57,0 & Breast & $\mathrm{F}$ \\
\hline Patient 4 & 19,6 & 12,9 & 159,0 & 12,3 & 8,1 & 63,0 & Lungs & $\mathrm{F}$ \\
\hline Patient 5 & 26,4 & 17,4 & 300,0 & 17,2 & 5,8 & 55,0 & Breast & $\mathrm{F}$ \\
\hline Patient 6 & 20,4 & 13,4 & 177,0 & 13,2 & 7,6 & 40,0 & Breast & $\mathrm{F}$ \\
\hline Patient 7 & 25,4 & 16,8 & 197,0 & 11,8 & 8,5 & 48,0 & Breast & $\mathrm{F}$ \\
\hline Patient 8 & 22,6 & 14,9 & 260,0 & 17,4 & 5,7 & 65,0 & Prostate & $\mathrm{F}$ \\
\hline Patient 9 & 31,7 & 20,9 & 252,0 & 12,1 & 8,3 & 65,0 & Colon & $\mathrm{F}$ \\
\hline Patient 10 & 26,7 & 17,6 & 265,0 & 15,1 & 6,6 & 38,0 & Ovary & $\mathrm{F}$ \\
\hline Patient 11 & 51,1 & 34,0 & 214,0 & 6,3 & 15,9 & 75,0 & Sarcoma & $\mathrm{F}$ \\
\hline Patient 12 & 29,5 & 19,6 & 250,0 & 12,7 & 7,9 & 49,0 & Uterus & $\mathrm{F}$ \\
\hline Patient 13 & 37,4 & 24,9 & 214,0 & 8,6 & 11,6 & 62,0 & Kidney & $\mathrm{F}$ \\
\hline Patient 14 & 35,2 & 23,4 & 209,0 & 8,9 & 11,2 & 61,0 & Prostate & $\mathrm{F}$ \\
\hline Patient 15 & 43,2 & 28,8 & 187,0 & 6,5 & 15,4 & 36,0 & Breast & $\mathrm{F}$ \\
\hline Patient 16 & 32,4 & 21,6 & 357,0 & 16,5 & 6,0 & 35,0 & Ovary & $\mathrm{F}$ \\
\hline Patient 17 & 28,6 & 19,1 & 220,0 & 11,5 & 8,7 & 58,0 & Chondrobl. & $\mathrm{F}$ \\
\hline Patient 18 & 32,8 & 21,8 & 182,0 & 8,3 & 12,0 & 75,0 & Glioblast. & M \\
\hline Patient 19 & 36,1 & 24,0 & 258,0 & 10,7 & 9,3 & 45,0 & Uterus & $\mathrm{F}$ \\
\hline Patient 20 & 38,6 & 25,7 & 391,0 & 15,2 & 6,6 & 64,0 & Pancreas & $\mathrm{F}$ \\
\hline Patient 21 & 30,6 & 20,4 & 275,0 & 13,5 & 7,4 & 47,0 & Uterus & $\mathrm{M}$ \\
\hline Patient 22 & 31,4 & 20,9 & 373,0 & 17,8 & 5,6 & 42,0 & Liver & $\mathrm{F}$ \\
\hline Patient 23 & 25,9 & 17,3 & 186,0 & 10,8 & 9,3 & 49,0 & Glioblast. & $\mathrm{M}$ \\
\hline Patient 24 & 36,3 & 24,2 & 162,0 & 6,7 & 14,9 & 56,0 & Colon & $\mathrm{F}$ \\
\hline Patient 25 & 15,0 & 10,0 & 168,0 & 16,8 & 6,0 & 60,0 & Prostate & $\mathrm{F}$ \\
\hline Patient 26 & 38,0 & 25,3 & 439,0 & 17,3 & 5,8 & 45,0 & Breast & $\mathrm{F}$ \\
\hline Patient 27 & 25,7 & 17,1 & 703,0 & 41,1 & 2,4 & 65,0 & Colon & $\mathrm{F}$ \\
\hline Patient 28 & 21,5 & 14,3 & 179,0 & 12,5 & 8,0 & 41,0 & Pancreas & $\mathrm{F}$ \\
\hline
\end{tabular}

Table 3. Mean age, total LDH, isoform $A$ and percentual contribution of iso-A to total LDH cluster for 30 healthy volunteers $(h s)$ and 28 tumour-bearing patients $(t b) . \sigma$, denotes the standard deviation of isoform A for each group.

\begin{tabular}{lllll} 
& \multicolumn{1}{c}{$\boldsymbol{H} \boldsymbol{t} \boldsymbol{b}$} & Units \\
\cline { 2 - 5 } $\mathbf{N}$ & 30 & 29 & \\
$\bar{x}$ age & 33.1 & 54.5 & Yrs \\
& $\bar{x}$ Total LDH & 192.0 & 287.8 & $\mathrm{U} / \mathrm{L}$ \\
& $\bar{x}$ Iso A & 6.84 & 30.9 & $\mathrm{ng} / \mathrm{ml}$ \\
& $\bar{x}$ percentual contribution & 2.93 & 8.3 & $\%$ \\
& \multicolumn{1}{c}{$\sigma$} & 1.48 & 7.66 & $\mathrm{ng} / \mathrm{ml}$ \\
\hline
\end{tabular}

DISCUSSION

Given the fact that LDH-A can specifically enable fermentative neoplastic metabolism, plasma 
levels of this isoenzyme can conceivably increase in apparently healthy subjects going through the process of developing a microtumour or avascular neoplastic lesion. This increase in the fractional contribution of the isoenzyme may take place without pushing total LDH beyond the formal upper limit of the reference range. Such increment implies a "silent" or clinically imperceptible shift towards aerobic glycolysis, a type of non-exertional fermentative metabolism highly specific of neoplastic cells. Pathological increments in aerobic glycolysis can be thus detected, non-invasively, during the initial or subclinical phases of tumorigenesis. Mitochondrial injury, age-related anaemia, and several other factors contributing to a deteriorating "installed capacity" for cellular respiration, progressively develop as a function of age in apparently healthy humans ${ }^{37-40}$. A shift towards fermentative metabolism in order to meet functional demands -such as repeated or persistent infections, inflammation, tissue damage, toxaemia, etc.- increases the probability of tumorigenesis ${ }^{41-43}$.

Our findings in tumour bearing patients $(t b)$ placed the mean percentual contribution of iso-A to total $\mathrm{LDH}$ at a distance exceeding 3 standard deviations $(\sigma)$ from the mean percentual contribution of iso-A to total LDH in healthy subjects $(h s$ ), for an effect size of 3.7 (as per the formulation

$\left.\Theta=\bar{x}_{t b}-\bar{x}_{h s} / \sigma_{h s}\right)$.

An estimation of the magnitude of the increment of plasma LDH-A in tumour-bearing patients relative to healthy subjects seemed appropriate in this context ${ }^{39}$. At the beginning of the study, we assumed that even at relatively low values of $\mathbf{n}$ (for both the set of healthy subjects providing reference values and that of tumour-bearing patients) an effect size higher than 1.2 in the scale provided in Table 5 would disprove the null hypothesis $\left(\boldsymbol{H}_{\mathbf{0}}\right)$.

Table 4. Computation of the effect size using Glass' estimator delta ( $\Delta)$. The numerator consists of the difference between the mean percentual contributions of LDH-A for both groups, while the denominator is the standard deviation of the second, healthy group $\left(\mathbf{x}_{h s}\right)$.

\begin{tabular}{|c|c|c|}
\hline$\Theta=\bar{x}_{t b}-\bar{x}_{h s}$ & $\Theta=8.3-2.9$ & $\Theta=3.7$ \\
\hline $\bar{x}_{\text {hs }}$ & 1.5 & \\
\hline
\end{tabular}

Table 5. A scale of effect sizes and corresponding sample size requirements to achieve statistical significance based on the work of Sawilowsky et al. (40), Cohen et al. (41) and Glass (42). In the present study, the effect size was 3.7 -well above the higher value on the scale- denoting an extremely strong effect and probably hinting at the need for a wider (in the sense of age distribution) sample of healthy subjects. Also, see Study Limitations.

\begin{tabular}{lcl}
\hline Qualification & Effect size $(\Theta)$ & Sample size requirement \\
\hline Extremely small & 0.01 & $\mathrm{n} \sim 100.000$ \\
\hline
\end{tabular}




\begin{tabular}{lll}
\hline Small & 0.20 & $\mathrm{n} \sim 10.000$ \\
Medium & 0.50 & $\mathrm{n} \sim 5.000$ \\
Large & 0.80 & $\mathrm{n} \sim 500$ \\
Very large & 1.20 & $\mathrm{n} \sim 50$ \\
Extremely large & 2.00 & $\mathrm{n} \sim 10$ \\
\hline
\end{tabular}

\section{Study limitations}

Given the high sensitivity achieved by extracting references values from healthy individuals under 53 yrs of age, this type of measurement of LDH-A would require adjunct analyses to pinpoint the exact organic location(s) of the suspected incipient micro lesions. This study, designed as an early detection device, provides only the framework for the clinical assessment of incipient signs of pathological metabolism and should be followed up by further investigation on collateral testing that can mitigate any loss of specificity stemming from the sensitivity/specificity trade-off. Also, given that the resulting effect size is -quite literally- off the chart, a bigger and more representative population sample would be needed, ensuring higher certainty and robustness to our findings. Our preliminary measurements already show an uptrend in plasma LDH-A in positive correlation with the age of the healthy subjects. An estimation of the effect size in subsequent samples would have to correct for age-related increments, independent from neoplastic pathology, within tumour-bearing patients.

\section{CONCLUSION}

LDH-A's inherent specificity to neoplastic metabolism makes it a useful proxy for systemic deficiencies of oxidative phosphorylation and ultra-early cancer detection. Increments in the percentual contribution of isoform A to total plasma levels of lactic dehydrogenase (exceeding 2 standard deviations above the mean healthy concentrations) reveal a pathological shift towards fermentative metabolism at some level of the organism. Regular, systematic measurements of the isoform A of LDH could, therefore, be used as an ultra-early biomarker of tumorigenesis.

\section{CONFLICTS OF INTEREST}

As of this writing, the authors have no conflicts of interest, directly or indirectly, by ownership or by affiliation with any brand, company or institution.

\section{REFERENCES}

1. Lactate Dehydrogenase A in Cancer: A promising target for diagnosis and therapy. Ping Miao Shile Sheng Xiaoguang Sun Jianjun Liu Gang Huang. IUBMB LIFE, 2013

2. Lactate dehydrogenase as a prognostic factor for survival time of terminally ill cancer patients: a preliminary study. Suh, S. Y. and Ahn, H. Y. (2007) Eur. J Cancer 
3. Lactate dehydrogenase A: A key player in carcinogenesis and potential target in cancer therapy. Yangbo Feng, Yanlu Xiong, Tianyun Qiao, Xiaofei Li, Lintao Jia, and Yong Han

4. Prognostic and predictive role of lactate dehydrogenase 5 expression in colorectal cancer patients treated with PTK787/ZK 222584 (vatalanib) antiangiogenic therapy. Koukourakis, M. I., Giatromanolaki, A., Sivridis, E., Gatter, K. C., Trarbach, T., et al. (2011) Clin. Cancer Res.

5. Lactate dehydrogenase $\mathrm{A}$ is overexpressed in pancreatic cancer and promotes the growth of pancreatic cancer cells. Rong, Y., Wu, W., Ni, X., Kuang, T., Jin, D., et al. (2013) Tumour Biol.

6. Understanding the Warburg effect: the metabolic requirements of cell proliferation. Matthew G Vander Heiden, Lewis C Cantley, Craig B Thompson Science 2009 May 22;324(5930):1029-33. doi: 10.1126/science.1160809.

7. Links between metabolism and cancer. Dang CV. Genes Dev. 2012;26(9):877-90

8. Hallmarks of cancer: the next generation. Hanahan, D., and Weinberg, R. A. (2011) Cell 144, 646- 674

9. Ultrastructural mitochondrial pathology in human astrocytic tumors: potentials implications pro-therapeutics strategies. Gabriel J. Arismendi-Morillo, Alan V. Castellano-Ramirez. Journal of Electron Microscopy

10. Quantifying intracellular rates of glycolytic and oxidative ATP production and consumption using extracellular flux measurements. Shona A. Mookerjee, Akos A. Gerencser, David G. Nicholls, Martin D. Brand J Biol Chem. 2017 Apr 28; 292(17): 7189-7207. doi: 10.1074/jbc.M116.774471

11. Inhibition of the Warburg effect with a natural compound reveals a novel measurement for determining the metastatic potential of breast cancers. Ritu Arora, David Schmitt, Balasubramanyam Karanam, Ming Tan, Clayton Yates, Windy Dean-Colomb Oncotarget. 2015 Jan 20;6(2):662-78. doi: 10.18632/oncotarget.2689.

12. Mitoenergetic Dysfunction Triggers a Rapid Compensatory Increase in Steady-State Glucose Flux. Dania C Liemburg-Apers, Tom J J Schirris, Frans G M Russel, Peter H G M Willems, Werner J H Koopman. Biophys J. 2015 Oct 6;109(7):1372-86. doi: 10.1016/j.bpj.2015.08.002.

13. Lactate dehydrogenase isoenzymes. Maekawa M. J Chromatogr. 1988;429:373-398.

14. Lactic Dehydrogenase In Human Neoplastic Tissues. Goldman RD, Kaplan NO, Hall TC. Cancer Res. 1964;24:389-399. 
15. Attenuation of LDH-A expression uncovers a link between glycolysis, mitochondrial physiology, and tumor maintenance. Fantin VR, St-Pierre J, Leder P. Cancer Cell. 2006;9:425-434.

16. Tumor markers in clinical practice: General principles and guidelines. S. SharmaIndian J Med Paediatr Oncol. 2009 Jan-Mar; 30(1): 1-8. doi: 10.4103/0971-5851.56328

17. Tumor markers: Past, present, and future. Diamandis EP. In: Tumor markers: Physiology, pathobiology, technology, and clinical applications. Washington DC: AACC Press; 2002. pp. 3-8.

18. Lactate Dehydrogenases as Metabolic Link between Tumor and Stroma in the Tumor Microenvironment. Deepshikha Mishra, Debabrata Banerjee Cancers (Basel). 2019 doi: 10.3390/cancers11060750

19. PRINCIPIA METABOLICA: Fundamentos Científicos y Clínicos para una Terapia Metabólica del Cáncer. Prieto Gratacós, E. vol.5./69 cuartavía trans ${ }^{\text {MEDIA }}$ ISBN 978987-42-4880-0. 2017

20. The Regulation and Function of Lactate Dehydrogenase A: Therapeutic Potential in Brain Tumor. Brain Pathol. Valvona C.J., Fillmore H.L., Nunn P.B., Pilkington G.J.2016;26:3-17. doi: 10.1111/bpa.12299.

21. Hypoxia-inducible proteins HIF $1 \alpha$ and lactate dehydrogenase LDH5, key markers of anaerobic metabolism, relate with stem cell markers and poor post-radiotherapy outcome in bladder cancer. Koukourakis M.I., Kakouratos C., Kalamida D., Bampali Z., Mavropoulou S., Sivridis E., Giatromanolaki A. Int. J. Radiat. Biol. 2016;92:353363. doi: 10.3109/09553002.2016.1162921.

22. FOXM1-LDHA signaling promoted gastric cancer glycolytic phenotype and progression. Jiang W., Zhou F., Li N., Li Q., Wang L. Int. J. Clin. Exp. Pathol. 2015;6:6756-6763.

23. Determinación de lactato deshidrogenasa en suero, plasma y líquido cefalorraquídeo. Wiener Laboratorio http://www.wienerlab.com.ar/VademecumDocumentos/Vademecum\%20espanol/ldh_1_sp.pdf

24. A history of the study of solid tumour growth: The contribution of mathematical modelling. R. P. Araujo, D. L. S. McElwain. Bulletin of Mathematical Biology

25. Onset of mortality increase with age and age trajectories of mortality | Josef Dolejs, Petra Marešová Clinical Interventions in Aging DOI https://doi.org/10.2147/CIA.S119327

26. Deciphering death: a commentary on Gompertz (1825) 'On the nature of the function expressive of the law of human mortality, and on a new mode of determining the value 
of life contingencies' Thomas B. L. Kirkwood. Philos Trans R Soc Lond B Biol Sci. 2015

27. The Law of Mortality Revisited: Interspecies Comparisons of Mortality S J Olshansky. J Comp Pathol. 2010 Jan;142 doi: 0.1016/j.jcpa.2009.10.016. Epub 2009

28. Rate of Aging, Rate of Dying and the Mechanism of Mortality. A C Economos. Arch Gerontol Geriatr. 1982 May;1(1):3-27. doi: 10.1016/0167-4943(82)90003-6.

29. Statistical Methods for Meta-Analysis. Larry V. Hedges \& Ingram Olkin (1985). Orlando: Academic Press. ISBN 978-0-12-336380-0.

30. Statistical Methods in Education \& Psychology, Third Edition. Glass, Gene V \& Hopkins, Kenneth D. (1996). Boston: Allyn \& Bacon.

31. Conceptual frameworks for mathematical modeling of tumor growth dynamics. Z̆.BajzerM.MarušićS.Vuk-Pavlović Mathematical and Computer Modelling Volume 23, Issue 6, March 1996, Pages 31-46

32. A mathematical model of tumor growth and its response to single irradiation. Yoichi Watanabe, Erik L. Dahlman, Kevin Z. Leder, and Susanta K. Hui Theor Biol Med Model. 2016 doi: 10.1186/s12976-016-0032-7

33. Mathematical Modeling of Tumor Growth Kinetics. Ž. Bajzer, S. Vuk-Pavlović, M. Huzak Mayo Clinic and Mayo Foundation Rochester, MN, USA https://link.springer.com/chapter/10.1007/978-0-8176-8119-7_3

34. Advances in mortality forecasting. Fanny Janssen 2018 Genus doi: 10.1186/s41118018-0045-7

35. Modelling and forecasting mortality. Pascariu, Marius. Ph.D. Thesis 2018 University of Southern Denmark Unit of Epidemiology, Biostatistics and Biodemography

36. Perspectives on Mortality Forecasting. Bengtsson T, Keilman N. 2014. Swedish National Social Insurance Board

37. Anemia in the elderly: clinical implications and new therapeutic concepts. Reinhard Stauder, Swee Lay Thein Haematologica. 2014 doi: 10.3324/haematol.2014.109967

38. Impact of anemia on hospitalization and mortality in older adults. Culleton BF, Manns BJ, Zhang J, Tonelli M, Klarenbach S, Hemmelgarn BR. Blood. 2006

39. Cancer as a mitochondrial metabolic disease. Thomas N. Seyfried Front Cell Dev Biol. 2015 doi: 10.3389/fcell.2015.00043

40. Mitochondria: the ultimate tumor suppressor, in "Cancer as a Metabolic Disease: On the Origin, Management, and Prevention of Cancer." Seyfried T. N. (2012d). (Hoboken, NJ: John Wiley \& Sons), 195-205

41. The paradox of cancer genes in non-malignant conditions: implications for precision medicine. Jacob J. Adashek, Shumei Kato, Scott M. Lippman, Razelle Kurzrock. 
Genome

Medicine volume 12,

Article number: 16 (2020)

https://doi.org/10.1186/s13073-020-0714-y

42. The living state and cancer. Albert Szent-Gyorgyi Proc. Natl. Acad. Sci. USA Vol. 74, No. 7, pp. 2844-2847, July 1977 Biophysics

43. Life-Cycling of Cancer: New Concept. Marina Shaduri, Marc Bouchoucha doi:10.5772/55385

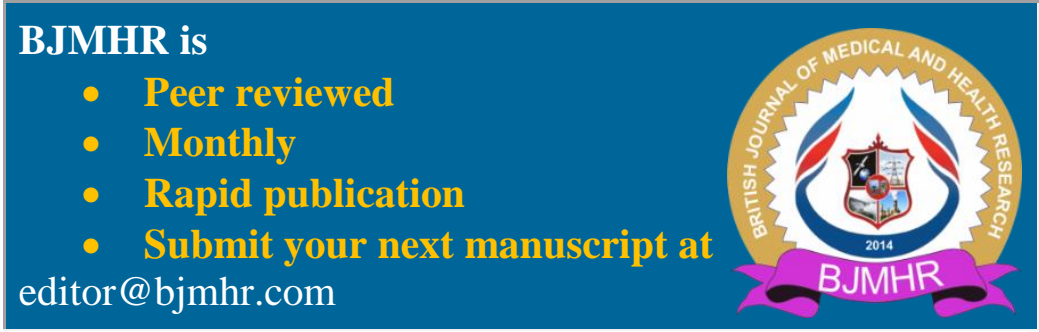

